\title{
Characterization of Arithmetical Equivalence of Number Fields by Galois Groups with Restricted Ramification
}

\author{
Mitsul TOHKAILIN and Manabu OZAKI
}

Kinki University and Waseda University

\begin{abstract}
We will give a characterization of arithmetical equivalence of number fields in terms of certain associated families of Galois groups with restricted ramification.
\end{abstract}

\section{Introduction}

The Dedekind zeta function $\zeta_{K}(s)$ is one of the most fundamental objects associated to a number field $K$. We believe that $\zeta_{K}(s)$ knows almost all the arithmetic properties of $K$. However, $\zeta_{K}(s)$ cannot identify completely the isomorphism class of the number field $K$ in general; For number fields $K$ and $K^{\prime}$, we say that $K$ and $K^{\prime}$ are arithmetically equivalent, denoting by $K \approx K^{\prime}$, if and only if the equality $\zeta_{K}(s)=\zeta_{K^{\prime}}(s)$ holds. Obviously, if $K \simeq K^{\prime}$ then $K \approx K^{\prime}$, but the converse does not hold in general. For example, $K=\mathbf{Q}(\sqrt[8]{3})$ and $K^{\prime}=\mathbf{Q}(\sqrt[8]{48})$ are arithmetically equivalent, but $K \not K^{\prime}$ (See [2], P. 86, $\left.(1,9)\right)$.

Therefore it is a basic problem to determine when two number fields $K$ and $K^{\prime}$ are arithmetically equivalent. The aim of the present paper is to give a characterization of arithmetical equivalence of number fields in terms of certain associated Galois groups with restricted ramification. Such an attempt was first made by N. Adachi and K. Komatsu in [1]: For any number field $F$ and prime number $p$, let $F_{\infty}(p)$ be the cyclotomic $\mathbf{Z}_{p}$-extension of $F\left(\zeta_{p}+\zeta_{p}^{-1}\right), \zeta_{p}$ being a primitive $p$-th root of unity, and denote by $X_{F_{\infty}(p),\{p\}}(p)$ the Galois group of the maximal abelian pro- $p$-extension over $F_{\infty}(p)$ unramified outside $p . X_{F_{\infty}(p),\{p\}}(p)$ has a natural $\Lambda_{p}:=\mathbf{Z}_{p}\left[\left[\operatorname{Gal}\left(\mathbf{Q}_{\infty}(p) / \mathbf{Q}\right)\right]\right]$-module structure as usual if $F \cap \mathbf{Q}_{\infty}(p)=\mathbf{Q}$, which holds for all but finitely many prime numbers $p$, via isomorphism $\operatorname{Gal}\left(F_{\infty}(p) / F\right) \simeq \operatorname{Gal}\left(\mathbf{Q}_{\infty}(p) / \mathbf{Q}\right)$ induced by the restriction.

THEOREM (Adachi-Komatsu [1]). Let $K$ and $K^{\prime}$ be totally real number fields. Then the followings are equivalent:

Received April 24, 2012; revised September 26, 2012

Mathematics Subject Classification: 11R42

Key words and phrases: arithmetical equivalence

This research was partially supported by JSPS, the Grant-in-Aid for Scientific Research (C) 21540030, and Waseda University, Grant for Special Research Projects 2012A-030. 
(1) $K \approx K^{\prime}$,

(2) $X_{K_{\infty}(p),\{p\}}(p) \simeq X_{K_{\infty}^{\prime}(p),\{p\}}(p)$ as $\Lambda_{p}$-modules for all but finitely many prime numbers $p$.

It is not clear whether we can omit the assumption "totally real" in the above theorem, because the proof of "(2) $\Longrightarrow(1)$ " largely relies on the Iwasawa main conjecture for totally real number fields, which was established by A. Wiles.

Our main result gives a characterization of arithmetical equivalence of any number fields in terms of a family of rather "small" Galois groups with restricted ramification, which are finite abelian groups.

\section{Main theorem}

In what follows, we will fix an algebraic closure of $\mathbf{Q}$ and regard any number fields and their algebraic extensions as subfields of it. For any set $S$ of prime numbers and number field $F$, we denote by $M_{F, S}$ the maximal abelian extension over $F$ unramified outside $S$, and let $X_{F, S}:=\operatorname{Gal}\left(M_{F, S} / F\right)$.

Our main result is the following:

THEOREM 1. For number fields $K$ and $K^{\prime}$ of finite degree, the following three statements are equivalent:

(1) $K \approx K^{\prime}$

(2) There exists a prime number $l_{0}$ such that

(i) $l_{0} \nmid[N: \mathbf{Q}]$ for the minimal Galois extension $N / \mathbf{Q}$ containing $K$ and $K^{\prime}$,

(ii) $l_{0}$ does not divide the class numbers of $K$ and $K^{\prime}$,

(iii) $l_{0}$ is unramified in $K / \mathbf{Q}$ and $K^{\prime} / \mathbf{Q}$,

(iv) $X_{K,\{p\}} / l_{0} \simeq X_{K^{\prime},\{p\}} / l_{0}$ for all but finitely many prime numbers $p$.

(3) Let $N / \mathbf{Q}$ be the minimal Galois extension containing both of $K$ and $K^{\prime}$. Then for any set $S$ of prime numbers and prime number $l$ satisfying $l \nmid[N: \mathbf{Q}]$, we have $X_{K, S}(l) \simeq X_{K^{\prime}, S}(l)$, where $X_{K, S}(l)$ and $X_{K^{\prime}, S}(l)$ denote the l-parts of $X_{K, S}$ and $X_{K^{\prime}, S}$, respectively.

\section{Preliminary lemmas}

In this section, we will give a collection of preliminary lemmas to prove Theorem 1.

The following lemma is well known:

LEMMA 1. Let $L / K$ be a finite extension of number fields and $N / K$ a Galois extension containing L. Put $G=\operatorname{Gal}(N / K), H=\operatorname{Gal}(N / L)$. For any prime ideal $\mathfrak{p}$ of $K$, we denote by $P_{\mathfrak{p}}$ the set of the prime ideals of L lying above $\mathfrak{p}$. Also, let $\mathfrak{P}$ be a prime ideal of $N$ lying above $\mathfrak{p}$ and $G_{\mathfrak{P}}$ the decomposition subgroup group of $G$ for $\mathfrak{P}$. Then the map

$$
\begin{aligned}
& H \backslash G / G_{\mathfrak{P}} \longrightarrow P_{\mathfrak{p}} \\
& H \sigma G_{\mathfrak{P}} \longmapsto \sigma \mathfrak{P} \cap L
\end{aligned}
$$


is a bijection. In the case where $\mathfrak{p}$ is unramified in $N / K$, we find especially the number of the prime ideals of $L$ lying over $\mathfrak{p}$ depends only on the Frobenius class of $\mathfrak{p}$ in $G$, namely, the conjugacy class of the Frobenius automorphism $\left[\frac{N / K}{\mathfrak{P}}\right]$ for $\mathfrak{P}$.

LEMMA 2. For a prime power $r$, we denote by $\mathbf{F}_{r}$ the finite field of order $r$. If a prime number $l$ and a positive integer $d$ satisfy $l \nmid d$ and $l \mid r-1$, then we have

$$
\left(\mathbf{F}_{r^{d}}^{\times}\right)^{l} \cap \mathbf{F}_{r}^{\times}=\left(\mathbf{F}_{r}^{\times}\right)^{l} .
$$

PROOF. Because the inclusion $\left(\mathbf{F}_{r^{d}}^{\times}\right)^{l} \cap \mathbf{F}_{r}^{\times} \supseteq\left(\mathbf{F}_{r}^{\times}\right)^{l}$ clearly holds, it is enough to show that the converse inclusion $\left(\mathbf{F}_{r^{d}}^{\times}\right)^{l} \cap \mathbf{F}_{r}^{\times} \subseteq\left(\mathbf{F}_{r}^{\times}\right)^{l}$ holds. Assume that $x \in \mathbf{F}_{r^{d}}^{\times}$and $x^{l} \in \mathbf{F}_{r}^{\times}$. Then we have $\left(x^{r-1}\right)^{l}=x^{l(r-1)}=1$. Suppose that the order $\operatorname{ord}\left(x^{r-1}\right)$ of $x^{r-1} \in \mathbf{F}_{r^{d}}^{\times}$is equal to $l$. Then it follows from $x^{(r-1) \cdot \frac{r^{d}-1}{r-1}}=x^{r^{d}-1}=1$ that $l \mid \frac{r^{d}-1}{r-1}$. However, this is impossible by $\frac{r^{d}-1}{r-1}=r^{d-1}+r^{d-2}+\cdots+r+1 \equiv d \not \equiv 0(\bmod l)$. Therefore we conclude that $x^{r-1}=1$, which implies $x \in \mathbf{F}_{r}$.

LEMMA 3. Let $G$ be a finite group and $p$ a prime number with $p \nmid \# G$. Assume that two subgroups $H_{1}, H_{2} \subseteq G$ satisfy

$$
\left.\mathbf{Q}_{p}[G] \underset{\mathbf{Q}_{p}\left[H_{1}\right]}{\otimes} \mathbf{Q}_{p} \simeq \mathbf{Q}_{p}[G] \underset{\mathbf{Q}_{p}\left[H_{2}\right]}{\otimes} \mathbf{Q}_{p} \text { (as } \mathbf{Q}_{p}[G] \text {-modules }\right) .
$$

Here we regard $\mathbf{Q}_{p}[G]$ as a two-sided $\mathbf{Q}_{p}[G]$-module via the ring structure of $i$, and the action of $G$ on $\mathbf{Q}_{p}[G] \underset{\mathbf{Q}_{p}\left[H_{i}\right]}{\otimes} \mathbf{Q}_{p}$ is defined by $\sigma(a \otimes b)=\sigma a \otimes b\left(a \in \mathbf{Q}_{p}[G], b \in \mathbf{Q}_{p}, \sigma \in\right.$ $G)$. Then for any $\mathbf{Z}_{p}[G]$-module $M$, we have

$$
M_{H_{1}} \simeq M_{H_{2}}\left(\text { as } \mathbf{Z}_{p} \text {-modules }\right)
$$

where $M_{H_{i}}=M / \sum_{\sigma \in H_{i}}(\sigma-1) M$ is the $H_{i}$-coinvariant of $M$.

PROOF. We recall the following lemma from the theory of integral representations of finite groups to prove Lemma 3:

LEMMA 4 ([2, p.626, $(30,16)])$. Let $G$ be a finite group and $p$ a prime number with $p \nmid \# G$. For any finitely generated $\mathbf{Z}_{p}[G]$-modules $A$ and $B$ without non-trivial $\mathbf{Z}_{p}$-torsions, $A \underset{\mathbf{Z}_{p}}{\otimes} \mathbf{Q}_{p} \simeq B \underset{\mathbf{Z}_{p}}{\otimes} \mathbf{Q}_{p}$ as $\mathbf{Q}_{p}[G]$-modules implies $A \simeq B$ as $\mathbf{Z}_{p}[G]$-modules.

It follows from

$$
\left(\mathbf{Z}_{p}[G] \underset{\mathbf{Z}_{p}\left[H_{i}\right]}{\otimes} \mathbf{Z}_{p}\right) \underset{\mathbf{Z}_{p}}{\otimes} \mathbf{Q}_{p} \simeq \mathbf{Q}_{p}[G] \underset{\mathbf{Q}_{p}\left[H_{i}\right]}{\otimes} \mathbf{Q}_{p} \text { (as } \mathbf{Q}_{p}[G] \text {-modules) }(i=1,2),
$$

and our assumption

$$
\left.\mathbf{Q}_{p}[G] \underset{\mathbf{Q}_{p}\left[H_{1}\right]}{\otimes} \mathbf{Q}_{p} \simeq \mathbf{Q}_{p}[G] \underset{\mathbf{Q}_{p}\left[H_{2}\right]}{\otimes} \mathbf{Q}_{p} \text { (as } \mathbf{Q}_{p}[G] \text {-modules }\right),
$$


that

$$
\mathbf{Z}_{p}[G] \underset{\mathbf{Z}_{p}\left[H_{1}\right]}{\otimes} \mathbf{Z}_{p} \simeq \mathbf{Z}_{p}[G] \underset{\mathbf{Z}_{p}\left[H_{2}\right]}{\otimes} \mathbf{Z}_{p} \text { (as } \mathbf{Z}_{p}[G] \text {-modules) }
$$

by using Lemma 4 . Hence we obtain

$$
M \underset{\mathbf{Z}_{p}[G]}{\otimes}\left(\mathbf{Z}_{p}[G] \underset{\mathbf{Z}_{p}\left[H_{1}\right]}{\otimes} \mathbf{Z}_{p}\right) \simeq M \underset{\mathbf{Z}_{p}[G]}{\otimes}\left(\mathbf{Z}_{p}[G] \underset{\mathbf{Z}_{p}\left[H_{2}\right]}{\otimes} \mathbf{Z}_{p}\right)
$$

as $\mathbf{Z}_{p}$-modules, where we define the right action of $\sigma \in G$ on $m \in M$ by $m \sigma:=\sigma^{-1} m$ to give a right $\mathbf{Z}_{p}[G]$-module structure to $M$.

On the other hand, by the associative law of tensor product, we have

$$
M \underset{\mathbf{z}_{p}[G]}{\otimes}\left(\mathbf{Z}_{p}[G] \underset{\mathbf{z}_{p}\left[H_{i}\right]}{\otimes} \mathbf{z}_{p}\right) \simeq\left(M \underset{\mathbf{z}_{p}[G]}{\otimes} \mathbf{Z}_{p}[G]\right) \underset{\mathbf{z}_{p}\left[H_{i}\right]}{\otimes} \mathbf{z}_{p} \simeq M \underset{\mathbf{z}_{p}\left[H_{i}\right]}{\otimes} \mathbf{z}_{p} \simeq M_{H_{i}}
$$

as $\mathbf{Z}_{p}$-modules for $i=1,2$, where we regard $M \underset{\mathbf{Z}_{p}[G]}{\otimes} \mathbf{Z}_{p}[G]$ as a right $H_{i}$-module via $(m \otimes$ $\alpha) h:=m \otimes \alpha h$ for $m \in M, \alpha \in \mathbf{Z}_{p}[G], h \in H_{i}$. Therefore it follows from (1) that $M_{H_{1}} \simeq$ $M_{H_{2}}$ as $\mathbf{Z}_{p}$-modules.

The following is the key to derive the equivalence of the three statements in Theorem 1:

Lemma 5 [3, P. 77, (1.3)]. Let $K$ and $K^{\prime}$ be any number fields. Then the following three statements are equivalent:

(1) $K \approx K^{\prime}$

(2) For a prime number $p$, let $g_{p}$ and $g_{p}^{\prime}$ be the numbers of the prime divisors of $K$ and $K^{\prime}$ lying over $p$, respectively. Then $g_{p}=g_{p}^{\prime}$ for all but finitely many prime numbers $p$.

(3) For any Galois extension $N / \mathbf{Q}$ containing $K$ and $K^{\prime}$, put $G=\operatorname{Gal}(N / \mathbf{Q}), H=$ $\operatorname{Gal}(N / K), H^{\prime}=\operatorname{Gal}\left(N / K^{\prime}\right)$. Then $\mathbf{Q}[G] \underset{\mathbf{Q}[H]}{\otimes} \mathbf{Q} \simeq \mathbf{Q}[G] \underset{\mathbf{Q}\left[H^{\prime}\right]}{\otimes} \mathbf{Q}$ as $\mathbf{Q}[G]-$ modules.

LeMma 6 ([4, p.41, Thm. 2.3.15]). Let $G$ be a pro-finite group and $N \subseteq G$ a pro- $p$ normal subgroup. Assume that the quotient group $Q=G / N$ has no non-trivial pro-psubgroups. Then the natural exact sequence

$$
1 \longrightarrow N \longrightarrow G \longrightarrow Q \longrightarrow 1
$$

splits.

\section{Proof of Theorem 1}

Proof. First, we will derive statement (1) from statement (2).

Assume that the prime number $l_{0}$ satisfies the condition given in statement (2) of the theorem. For a prime number $q$ and $n \geq 0$, we write $K_{q^{n}}$ for the ray class field of modulo 
$q^{n} \mathcal{O}_{K}$ over $K, \mathcal{O}_{K}$ being the integer ring of $K$. Then $M_{K,\{q\}}=\bigcup_{n=1}^{\infty} K_{q^{n}}$, and $X_{K,\{q\}}=$ $\underset{n}{\lim } \operatorname{Gal}\left(K_{q^{n}} / K\right)$. We denote by $I_{K, q}$ the group of the fractional ideals of $K$ which are prime to $q$, and we write $P_{K, q}$ for the group of the principal ideals contained in $I_{K, q}$. Also, we put $S_{K, q^{n}}=\left\{\alpha \mathcal{O}_{K} \mid \alpha \in K^{\times}, \alpha \equiv 1\left(\bmod q^{n}\right)\right\}$.

If we assume $q \neq l_{0}$, then, by using class field theory, we have

$$
X_{K,\{q\}} / l_{0} \simeq \operatorname{Gal}\left(K_{q} / K\right) / l_{0} \simeq\left(I_{K, q} / S_{K, q}\right) / l_{0} \simeq\left(P_{K, q} / S_{K, q}\right) / l_{0}
$$

because $\operatorname{Gal}\left(K_{q^{n}} / K_{q}\right)$ is a $q$-group $\left(q \neq l_{0}\right)$ and the $l_{0}$-part of the class group of $K$, which is isomorphic to that of $I_{K, q} / P_{K, q}$, is trivial from our assumption. Thus we derive from the exact sequence

$$
\mathcal{O}_{K}^{\times} \longrightarrow\left(\mathcal{O}_{K} / q\right)^{\times} \longrightarrow P_{K, q} / S_{K, q} \longrightarrow 0
$$

and (2) the exact sequence

$$
\mathcal{O}_{K}^{\times} \stackrel{\pi_{K, q}}{\longrightarrow}\left(\mathcal{O}_{K} / q\right)^{\times} / l_{0} \longrightarrow X_{K,\{q\}} / l_{0} \longrightarrow 0 .
$$

Assume that the prime $q$ is unramified in $K / \mathbf{Q}$ and let $q \mathcal{O}_{K}=\mathfrak{q}_{1} \cdots \mathfrak{q}_{g_{q}}$ be the prime decomposition in $K$. Then it follows from the Chinese Remainder Theorem that

$$
\left(\mathcal{O}_{K} / q\right)^{\times} / l_{0} \simeq \bigoplus_{i=1}^{g_{q}}\left(\mathcal{O}_{K} / \mathfrak{q}_{i}\right)^{\times} / l_{0}
$$

If we further assume that $q \equiv 1\left(\bmod l_{0}\right)$, then

$$
\left(\mathcal{O}_{K} / q\right)^{\times} / l_{0} \simeq \bigoplus_{i=1}^{g_{q}}\left(\mathcal{O}_{K} / \mathfrak{q}_{i}\right)^{\times} / l_{0} \simeq\left(\mathbf{Z} / l_{0}\right)^{\oplus g_{q}}
$$

Recall that $N / \mathbf{Q}$ denotes the minimal Galois extension containing $K$ and $K^{\prime}$. Put $L=N\left(\mu_{l_{0}},\left\{\sqrt[l_{0}]{\varepsilon} \mid \varepsilon \in \mathcal{O}_{N}^{\times}\right\}\right), G_{N}=\operatorname{Gal}(N / \mathbf{Q}), G_{N\left(\mu_{0}\right)}=\operatorname{Gal}\left(N\left(\mu_{l_{0}}\right) / \mathbf{Q}\right)$, and $G_{L}=\operatorname{Gal}(L / \mathbf{Q})$, where $\mu_{l_{0}}$ stands for the group of the $l_{0}$-th roots of unity. We note that [L:N( $\left.\left.N \mu_{0}\right)\right]=l_{0}^{e}$ for some $e \geq 0$, and that $\left[N\left(\mu_{l_{0}}\right): \mathbf{Q}\right]$ is prime to $l_{0}$ from our assumption. For any Galois extension $F / \mathbf{Q}$ and prime number $q$ which is unramified in $F$, we set $C_{F / \mathbf{Q}}(q)=\left\{\left[\frac{F / \mathbf{Q}}{\mathfrak{q}}\right]|\mathfrak{q}| q\right.$ is a prime of $\left.F\right\}$, and for any group $G$ and $\sigma \in G$, put $C(G, \sigma)=\left\{g \sigma g^{-1} \mid g \in G\right\}$.

In what follows, we will show that for any $\sigma \in G_{N}$ there exist infinitely many prime numbers $q$ satisfying the following three conditions:

(a) $q \equiv 1\left(\bmod l_{0}\right)$

(b) the prime $q$ is unramified in $L$ and $C_{N / \mathbf{Q}}(q)=C\left(G_{N}, \sigma\right)$,

(c) $\langle x\rangle \cap \operatorname{Gal}(L / N)=\{1\}$ for all $x \in C_{L / \mathbf{Q}}(q)$. 
Since $l_{0}$ is unramified in $N / \mathbf{Q}$ from our assumption on $l_{0}$, we have $N \cap \mathbf{Q}\left(\mu_{l_{0}}\right)=\mathbf{Q}$ and the isomorphism

$$
\begin{array}{ccc}
G_{N\left(\mu_{l_{0}}\right)} & \simeq G_{N} \times \operatorname{Gal}\left(\mathbf{Q}\left(\mu_{l_{0}}\right) / \mathbf{Q}\right) . \\
x & \mapsto\left(\left.x\right|_{N},\left.x\right|_{\mathbf{Q}\left(\mu_{l_{0}}\right)}\right)
\end{array}
$$

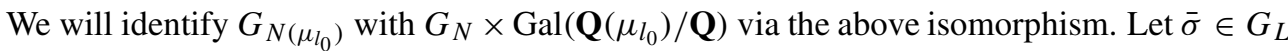
be an automorphism such that $\left.\bar{\sigma}\right|_{N\left(\mu_{0}\right)}=(\sigma, 1)$. Because $l_{0} \nmid \# G_{N}$ by our assumption on $l_{0}$, there exists a positive integer $d$ such that $d \equiv 1\left(\bmod \# G_{N}\right)$ and $d \equiv 0\left(\bmod l_{0}^{e}\right)$. Put $y=\bar{\sigma}^{d} \in G_{L}$. Then we see that $\left.y\right|_{N\left(\mu_{0}\right)}=\left(\sigma^{d}, 1^{d}\right)=(\sigma, 1)=\left.\bar{\sigma}\right|_{N\left(\mu_{0}\right)}$ and (ord $\left.y, l_{0}\right)=$ 1 since $l_{0}^{e} \| \# G_{L}$. By the Chebotarev density theorem, there exist infinitely many prime numbers $q$ such that $q$ is unramified in $L$ and $C_{L / \mathbf{Q}}(q)=C\left(G_{L}, y\right)$. We will show that this prime number $q$ satisfies conditions (a), (b), and (c).

For any subset $C$ of $G_{L}$, we put $\left.C\right|_{N}=\left\{\left.g\right|_{N} \in G_{N} \mid g \in C\right\}$. Then we have $C_{N / \mathbf{Q}}(q)=\left.C\left(G_{L}, y\right)\right|_{N}=C\left(G_{N},\left.y\right|_{N}\right)=C\left(G_{N}, \sigma\right)$, which implies that $q$ satisfies (b). Since $C_{\mathbf{Q}\left(\mu_{0}\right) / \mathbf{Q}}(q)=\left.C_{L / \mathbf{Q}}(q)\right|_{\mathbf{Q}\left(\mu_{l_{0}}\right)}=\left.C\left(G_{L}, y\right)\right|_{\mathbf{Q}\left(\mu_{l_{0}}\right)}=1$, we see that $\left(\frac{\mathbf{Q}\left(\mu_{l_{0}}\right) / \mathbf{Q}}{q}\right)=1$, which implies that $q$ satisfies (a). Finally, let $x \in C_{L / \mathbf{Q}}(q)$ and $g \in\langle x\rangle \cap \operatorname{Gal}(L / N)$ be any element. Since $x \in C_{L / Q}(q)=C\left(G_{L}, y\right)$, we find that $x=z y z^{-1}$ for some

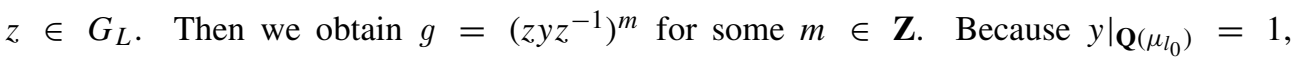

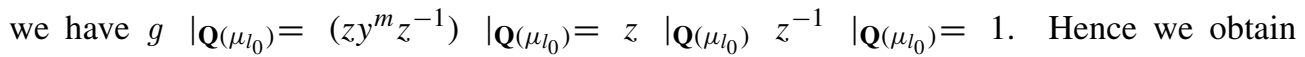
$g \in \operatorname{Gal}(L / N) \cap \operatorname{Gal}\left(L / \mathbf{Q}\left(\mu_{l_{0}}\right)\right)=\operatorname{Gal}\left(L / N\left(\mu_{l_{0}}\right)\right)$. It follows from the facts ord $g=$ ord $\left(z y z^{-1}\right)^{m}=$ ord $y^{m}$ and (ord $\left.y, l_{0}\right)=1$ that (ord $\left.g, l_{0}\right)=1$, which implies $g=1$ since $\operatorname{Gal}\left(L / N\left(\mu_{l_{0}}\right)\right)$ is an $l_{0}$-group. Hence we find that $q$ satisfies (c). Thus we conclude that $q$ satisfies conditions (a), (b) and (c).

Let $p$ be any prime number which is unramified in $N / \mathbf{Q}$. By the above discussion, there exist infinitely many prime numbers $q$ such that (a) $q \equiv 1\left(\bmod l_{0}\right),\left(\mathrm{b}^{\prime}\right) q$ is unramified in $L$ and $C_{N / \mathbf{Q}}(q)=C_{N / \mathbf{Q}}(p)$, and (c) $\langle x\rangle \cap \operatorname{Gal}(L / N)=\{1\}$ for all $x \in C_{L / \mathbf{Q}}(q)$. Furthermore, by our assumption, we may assume that the prime number $q$ satisfies condition (iv) of statement (2), that is, $X_{K,\{q\}} / l_{0} \simeq X_{K^{\prime},\{q\}} / l_{0}$. Condition (c) implies that all the prime ideals of $N$ lying over $q$ are completely decomposed in $L / N$. Hence, for any prime $\mathfrak{q}_{K}$ of $K$ lying over $q$ and a prime $\mathfrak{q}_{N}$ of $N$ lying over $\mathfrak{q}_{K}$, we obtain the inclusion and the isomorphism

$$
\left(\mathcal{O}_{K} / \mathfrak{q}_{K}\right)^{\times} \hookrightarrow\left(\mathcal{O}_{N} / \mathfrak{q}_{N}\right)^{\times} \simeq\left(\mathcal{O}_{L} / \mathfrak{Q}\right)^{\times}
$$

for a prime $\mathfrak{Q}$ of $L$ lying over $\mathfrak{q}_{N}$. We see that $l_{0} \nmid\left[\mathcal{O}_{N} / \mathfrak{q}_{N}: \mathcal{O}_{K} / \mathfrak{q}_{K}\right]$ from our assumption on $l_{0}$. Since $\left(\varepsilon \bmod \mathfrak{q}_{K}\right) \in\left(\mathcal{O}_{K} / \mathfrak{q}_{K}\right)^{\times} \operatorname{maps}$ to $\left(\left(\sqrt[l_{0}]{\varepsilon}\right)^{l_{0}} \bmod \mathfrak{Q}\right) \in\left(\left(\mathcal{O}_{L} / \mathfrak{Q}\right)^{\times}\right)^{l_{0}}$ for any $\varepsilon \in \mathcal{O}_{K}^{\times}$, it follows from Lemma 2 that $\left(\varepsilon \bmod \mathfrak{q}_{K}\right) \in\left(\left(\mathcal{O}_{K} / \mathfrak{q}_{K}\right)^{\times}\right)^{l_{0}}$ for any $\varepsilon \in \mathcal{O}_{K}^{\times}$, which implies $\pi_{K, q}\left(\mathcal{O}_{K}^{\times}\right)=0$, where $\pi_{K, q}$ is the map defined in (3). Therefore we derive from 
condition (a), (3) and (4) that

$$
X_{K,\{q\}} / l_{0} \simeq(\mathbf{Z} / l \mathbf{Z})^{\oplus g_{q}},
$$

where $g_{q}$ is the number of primes of $K$ lying over $q$. Similarly, we also have

$$
X_{K^{\prime},\{q\}} / l_{0} \simeq(\mathbf{Z} / l \mathbf{Z})^{\oplus g_{q}^{\prime}},
$$

where $g_{q}^{\prime}$ is a number of primes of $K^{\prime}$ lying over $q$. Therefore we see by using our assumption $X_{K,\{q\}} / l_{0} \simeq X_{K^{\prime},\{q\}} / l_{0}$ that $g_{q}=g_{q}^{\prime}$. On the other hand, it follows from property $\left(\mathrm{b}^{\prime}\right)$ of the prime $q$ and Lemma 1 that $g_{p}=g_{q}$ and $g_{p}^{\prime}=g_{q}^{\prime}$. Thus we have shown that $g_{p}=g_{p}^{\prime}$ for any prime number $p$ which is unramified in $N / \mathbf{Q}$. Therefore, by using Lemma $5((2) \Longrightarrow(1))$, we conclude $K \approx K^{\prime}$.

Next, we will derive statement (3) of the theorem assuming statement (1) holds.

Let $l$ be any prime number which satisfies $l \nmid[N: \mathbf{Q}]$. Since $l \nmid[N: K]$ and $M_{K, S}(l) / K$ is a pro-l-extension, we see $N \cap M_{K, S}(l)=K$. Put $F=N M_{K, S}(l)$ and $H=\operatorname{Gal}(N / K)$. We will show that $\operatorname{Gal}(F / N) \simeq\left(X_{N, S}(l)\right)_{H}$, where we regard $X_{N, S}(l)$ as a $\operatorname{Gal}(N / \mathbf{Q})$ module via inner automorphism of $\operatorname{Gal}\left(M_{N, S}(l) / \mathbf{Q}\right)$ induced by an extension of each element of $\operatorname{Gal}(N / \mathbf{Q})$ to $\operatorname{Gal}\left(M_{N, S}(l) / \mathbf{Q}\right)$.

Let $F^{\prime}$ be the intermediate field of $M_{N, S}(l) / N$ which satisfies $\operatorname{Gal}\left(F^{\prime} / N\right) \simeq\left(X_{N, S}(l)\right)_{H}$. It follows from Lemma 6 together with the fact that $H$ acts trivially on $\operatorname{Gal}\left(F^{\prime} / N\right)$ that $\mathrm{Gal}\left(F^{\prime} / K\right)$ admits a direct product decomposition

$$
\operatorname{Gal}\left(F^{\prime} / K\right)=\operatorname{Gal}\left(F^{\prime} / N\right) \times \operatorname{Gal}\left(F^{\prime} / E\right)
$$

for some sub-Galois-extension $E / K$ of $F^{\prime} / K$ with $\operatorname{Gal}\left(F^{\prime} / E\right) \simeq H$ and $\operatorname{Gal}(E / K) \simeq$ $\operatorname{Gal}\left(F^{\prime} / N\right)$. Since $\operatorname{Gal}(E / K)$ is a pro-l abelian group and the order of $\operatorname{Gal}\left(F^{\prime} / E\right)$ is prime to $l$, we see that $E \subseteq M_{K, S}(l)$. Hence $F^{\prime}=N E \subseteq N M_{K, S}(l)=F$. Conversely, because $F \subseteq M_{N, S}(l)$ and the action of $H$ on $\operatorname{Gal}(F / N)$ is trivial, we find that $F \subseteq F^{\prime}$. Thus we have shown $F=F^{\prime}$. Therefore we have

$$
\left(X_{N, S}(l)\right)_{H} \simeq \operatorname{Gal}(F / N) \simeq X_{K, S}(l),
$$

since $N \cap M_{K, S}(l)=K$. Similarly, we obtain

$$
\left(X_{N, S}(l)\right)_{H^{\prime}} \simeq X_{K^{\prime}, S}(l)
$$

for $H^{\prime}=\operatorname{Gal}\left(N / K^{\prime}\right)$. Then it follows from Lemma $5((1) \Longrightarrow(3))$ and Lemma 3 that

$$
X_{K, S}(l) \simeq\left(X_{N, S}(l)\right)_{H} \simeq\left(X_{N, S}(l)\right)_{H^{\prime}} \simeq X_{K^{\prime}, S}(l) .
$$

Thus we have shown that statement (3) holds.

Finally, it is obvious that statement (3) implies statement (2). This completes the proof of Theorem 1. 


\section{References}

[ 1 ] N. AdACHI and K. Komatsu, The maximal $p$-extensions and zeta-functions of algebraic number fields, Mem. School Sci. Engrg. Waseda Univ. No. 51 (1987), 25-31.

[ 2 ] C. W. CURTIS and I. REINER, Methods of representation theory Vol. I, John Wiley \& Sons, Inc., New York (1990).

[ 3 ] N. Klingen, Arithmetical Similarities, Clarendon Press, Oxford (1998).

[ 4 ] L. Ribes and P. Zaless KiI, Profinite Groups, A series of Modern surveys in Mathematics, vol. 40, Springer (1991).

Present Addresses:

Mitsul TOHKAILIN

DEPARTMENT OF MATHEMATICS,

FACULTY OF SCIENCE AND TECHNOLOGY,

KINKI UNIVERSITY,

3-4-1, KOWAKAE, HIGASHI-OSAKA, 577-8502 JAPAN.

e-mail: tohkailin@math.kindai.ac.jp

MANABU OZAKI

DEPARTMENT OF MATHEMATICS,

SCHOOL OF FUndaMENTAL SCIENCE AND ENGINEERING,

WASEDA UNIVERSITY,

3-4-1, OHKUBO, SHINJUKU-KU, TOKYO, 169-8555 JAPAN.

e-mail: ozaki@waseda.jp 